\title{
La construction du sens social en discours. Le cas de Grexit
}

\section{The building of social meaning in discourse. The case of Grexit}

\author{
Elżbieta Biardzka \\ Université de Wrocław \\ ebiardzka@wp.pl
}

\begin{abstract}
In this paper we analyse occurrences of the word Grexit in terms of syntagmatic and textual relations. We aim to show the semantic and pragma-discursive determinations of its social meaning, which appears to be vague and confounding. The study is based on the corpus of press texts published in Le Monde, Libération and Le Figaro between 6 July 2015 (the day after the Greek referendum) and 11 July 2015 (date of the agreement between Greece and its creditors).
\end{abstract}

Keywords: social meaning, Grexit, reformulations, designational paradigm.

\section{INTRODUCTION}

Le «non »grec au référendum du 5 juillet $2015^{1}$ a provoqué une importante production médiatique où on a vu apparaître, entre autres, la dénomination propre Grexit $^{2}$. Il s'agit en effet d'un néologisme anglais forgé en 2012 dans une étude économique de deux analystes du Citygroup, Willem H. Buiter et Ebrahim Rahbari (2012). Le Grexit est un mot-valise, amalgamant, selon ses auteurs-mêmes, le voca-

\footnotetext{
${ }^{1}$ Rappelons que les Grecs ont refusé d'accepter des mesures d'austérité imposées à la Grèce par les créanciers (proposition faite par l'UE, la BCE (Banque centrale européenne) et le FMI (Fonds monétaire international) le jeudi 25 juin 2015).

${ }^{2}$ Une recherche simple sur Google révèle plus de 3 millions d'occurrences en 0,44 seconde.
} 
ble Greek, l'abréviation EA (euro area) et exit [Greek EA exit ('Grexit') = littéralement, 'la sortie de la Grèce de la zone euro']. Le néologisme émerge immédiatement en discours médiatique mondial, car il colle congrûment à la matérialité du discours politique du moment. Rappelons qu'en 2012, Alexis Tsipras, le leader du parti d'extrême-gauche Syriza, alors opposé au gouvernement, déclare que la Grèce doit refuser les mesures d'austérité imposées par les institutions européennes, même si cela doit signifier la suspension des aides européennes promises, l'impossibilité de rembourser ses dettes et l'abandon de l'euro pour pouvoir émettre sa propre monnaie afin de payer les créanciers. Par la suite, l'ancien premier ministre Lucas Papademos évoque expressis verbis une sortie possible de la zone euro dans une interview accordée au Wall Street Journal. Le journal allemand Der Spiegel révèle également en 2015 qu'Angela Merkel juge inévitable une sortie de la Grèce de la zone euro si la gauche radicale prend le pouvoir.

Le Grexit s'apparente, par son sens discursif et surtout son fonctionnement, à ce qu'Alice Krieg-Planque appelle une «formule» (2003, 2006, 2009, 2010). Il présente ainsi un caractère stable, figé et se prête à l'inventivité lexicale. Son signifiant est très largement connu du public ${ }^{3}$. Il «n'appartient pas à un ordre de la réalité mondaine » mais « à l'ordre du réel discursif et symbolique » (Krieg-Planque, 2010, p. 11). En effet, la formule se caractérise toujours par un certain «flottement sémantique » qui permet aux locuteurs de se la réapproprier (Krieg-Planque, 2009, p. 75). La formule réfère donc pour tous à une réalité, parfois floue et/ou controversée, mais socialement partagée. Le Grexit nous intéressera comme une unité de sens opaque qui absorbe les enjeux économiques et sociaux indissolubles de la politique.

\section{CORPUS DE RECHERCHE, OBJECTIFS ET MÉTHODE DU TRAVAIL}

Le corpus de recherche est constitué de textes journalistiques du Monde, de Libération et du Figaro, publiés entre le 6 juillet 2015 (le lendemain du référendum grec) et le 11 juillet 2015 (l'accord entre la Grèce et ses créanciers). Le choix des dates n'est pas anodin. Le référendum en Grèce a marqué un moment critique dans la réalité économique et socio-politique mondiale. À ce moment, le danger de la sortie grecque de la zone euro devient imminent et constitue une sorte de déclencheur d'une production médiatique importante, qui est le milieu dans lequel se construit le sens social d'un nouveau phénomène. Il doit être nommé, expliqué à la communauté linguistique donnée puisqu'il constitue une rupture dramatique dans l'ordre «normal » des choses ( $c f$. Moirand, 2007 ; Veniard, 2013). De la sorte, les médias font un certain travail sémantique pour «donner du sens » au phénomène

\footnotetext{
${ }^{3} C f$. note 2 .
} 
nouveau : le Grexit devient un véritable mot-vedette, objet de discours : il se positionne dans les titres médiatiques, émerge dans les articles, s'accompagne de différentes explications in praesentia, entre dans des réseaux diaphoriques complexes, fonctionnant souvent en anaphore conceptuelle résomptive. Dans notre étude, nous nous intéresserons aux textes d'information et commentaires, qui diffusent du sens à une grande échelle et sont destinés au grand public. Le tableau ci-dessous montre le nombre d'occurrences du Grexit notées dans les articles analysés. Au total, en 5 jours, le vocable est apparu 161 fois dans 78 articles. Il a fait la « une » 5 fois dans Le Monde, 4 fois dans Le Figaro sans émerger à la première page dans Libération :

\begin{tabular}{|l|l|c|}
\hline \multicolumn{1}{|c|}{ Titre } & Date & $\begin{array}{c}\text { Occurrences } \\
\text { Grexit }\end{array}$ \\
\hline Le Monde & le 6 juillet & 8 \\
Le Figaro & & 7 \\
Libération & & 4 \\
\hline Le Monde & le 7 juillet & 22 \\
Libération & & 8 \\
Le Figaro & & 9 \\
\hline Le Monde & le 8 juillet & 16 \\
Libération & & 5 \\
Le Figaro & & 19 \\
\hline Le Monde & le 9 juillet & 23 \\
Libération & & 4 \\
Le Figaro & \multirow{2}{*}{10 juillet } & 16 \\
\hline Le Monde & & 11 \\
Libération & le 11 juillet & 0 \\
Le Figaro & & 0 \\
\hline Le Monde & 4 \\
Libération & & 1 \\
Le Figaro & & 4 \\
\hline
\end{tabular}

Sur la base de ce corpus, nous chercherons à découvrir la stratégie momentanée de la praxis linguistique et sociale adoptée par les médias d'information par rapport à la manière de nommer le réel. À cette fin, nous examinerons les occurrences du Grexit sur l'axe syntagmatique ainsi que ses reformulations in praesentia et sur l'axe textuel (paradigmatique) ce qui nous permettra d'examiner le paradigme désignationnel (Mortureux, 1993) défini comme une liste de syntagmes (en général nominaux) fonctionnant en coréférence avec un vocable initial (Grexit) dans un discours donné. Notre étude se situe ainsi dans la lignée des travaux de Patrick Seriot (1986), Marie-Françoise Mortureux (1993), Paul Siblot (1997; 2001), Sophie Moirand (2007), Alice Krieg-Planque (2009), Marie-Anne Paveau (2008), Laura Calabrese- 
Steimberg (2010), ou Marie Veniard (2013) qui s'intéressent à la nomination puisqu'elle participe à la construction du sens social de l'événement ${ }^{4}$.

Le langage qui se matérialise dans le récit médiatique sur la crise grecque joue sur le statut référentiel complexe et flou du Grexit (point 3.0.), se répercute sur l'expression de l'agentivité et des autres arguments sémantiques (point 4.1.). La multiplication des classes référentielles (points 4.2. et 4.3.) dans les reformulations du vocable empêche d'appréhender clairement son sens.

\section{STATUT RÉFÉRENTIEL ET PRAXIS DISCURSIVE DU GREXIT}

Par rapport au statut référentiel des autres dénominations examinées par l'analyse du discours récente ( $c f$. les travaux cités infra), telles Tchernobyl, Tienanmen, le 11 septembre ou la guerre d'Afghanistan, le statut référentiel du Grexit est assez spécial. En analyse du discours, la nomination est analysée souvent par rapport à ce qu'on a l'habitude d'appeler un « événement réel » (Moirand, 2007, p. 4 ; Veniard, 2013, pp. 7-12 et 15). Cependant, la particularité de l'événement auquel renvoie le vocable Grexit est qu'il n'a jamais eu d'existence dans le monde que nous tenons pour réel. Ainsi, le discours met en jeu la spécificité référentielle de cette dénomination propre qui repose sur une présupposition existentielle : tout ce à quoi on réfère doit exister (Searle, 1972, p. 121 ; Kleiber, 1997).

De la sorte, sur l'axe syntagmatique, le Grexit s'emploie rarement en tant que noyau du syntagme nominal. Si cela lui arrive tout de même, il apparaît dans le contexte de verbes soulignant son appartenance au monde possible, comme «envisager » en (7). Il ne se positionne pas non plus en fonction de complément de noms déverbaux classiques comme "événement» ou «crise», mais s'accompagne de dénominations telles que «perspective » (1), «hypothèse » (3), « spectre » (2) ou encore « scénario » (6), se qualifie de " probable » au moyen d'un verbe copule (4) et se laisse substituer (sur l'axe paradigmatique) par le nom « option » (5) :

(1) À ce stade, c'est plus la perspective d'un Grexit qui sape les fondations de l'édifice européen, c'est le poison de l'incertitude. (Le Figaro, 7/07/15, p. 1)

(2) Le spectre du Grexit se précise (...) (Le Figaro, 6/07/15, p. 1)

(3) De fait, ce résultat bascule le pays et la zone euro dans l'inconnu, tout en crédibilisant l'hypothèse d'un « Grexit » - une sortie de la Grèce de l'euro. (Le Monde, $7 / 07 / 15$, p. 7)

(4) Le « Grexit » est plus probable que jamais. (Le Monde, $7 / 07 / 15$, p. 1)

\footnotetext{
${ }^{4} C f$. « (...) la nomination est un acte de catégorisation, une praxis qui est simultanément sociale et linguistique » (Détrie, Siblot, Vérine, 2001, pp. 205-206), ainsi que «nommer, ce n'est pas seulement se situer à l'égard de l'objet, c'est aussi prendre position à l'égard d'autres dénominations du même objet, à travers lesquelles des locuteurs prennent également position » (Siblot, 1997, p. 55).
} 
(5) (...) la chancelière cherche encore à l'éviter, consciente des risques qu'une telle option ferait subir à la zone euro. (Le Figaro, 7/07/15, p. 3)

(6) Le scénario du «Grexit» redouté par les marchés (...) semblait prendre corps (...) (Le Monde, 7 /07/15, p. 7)

(7) Est-ce à dire que l'institution ${ }^{5}$ envisage, elle aussi «un Grexit»? (Le Monde, $7 / 07 / 15$, p. 7)

Au sens du potentiel il s'ajoute ensuite l'idée de l'incertain et du dangereux le Grexit est reformulé par « l'équation à plusieurs inconnues » (14), "saut dans l'inconnu » (16), « le compte à rebours » $(17$, infra $)$. Le Grexit est une chose à éviter (9), une chose qu'on ne souhaite pas (8) :

(8) « Personne ne veut casser de la porcelaine. La chancelière ne veut pas d'un Grexit (...)» (Le Monde, $8 / 07 / 15$, p. 2)

(9) (...) éviter le « Grexit » (Le Monde, 8/07/15, p. 1)

(10) (...) si la zone euro peut éventuellement se remettre d'un Grexit, l'UE n'a rien à gagner du chaos qui s'installerait dans ce pays des Balkans (...). (Libération, $8 / 07 / 15$, p. 15)

(11) (...) si Grexit il y aura (...) (Le Figaro, 7/07/15, p. 3)

(12) Un Grexit changerait les termes du débat. (Le Figaro, 7/07/15, p. 4)

(13) Un « Grexit » aurait de gigantesques coûts politiques (...) (Le Monde, 8 /07/15, p. 4)

(14) Après le référendum grec, l'équation à plusieurs inconnues. (Libération, $8 / 07 / 15$, p. 20)

Le statut potentiel et incertain du Grexit dans la praxis discursive se manifeste aussi par la contextualisation de la dénomination dans des structures conditionnelles comme en $(10,11)$ (« si la zone euro... »), l'emploi récurrent de l'article indéfini « un Grexit » $(10,12,13)$ (par rapport à « le Grexit ») et par l'emploi du conditionnel $(10,12,13)$ qui a une valeur hypothétique et non « journalistique » ou « évidentielle » ( $c f$. Dendale, 2001). L'incertain se réalise aussi sur le mode métaphorique : le « scénario » n'est que le déroulement de faits devant se réaliser selon une intrigue programmée (15) :

(15) (...) le scénario d'un « Grexit » (...) (Le Monde, 8/07/15, p. 2)

(16) (...) d'éviter le scénario du pire, ce «saut dans l'inconnu » (...) le scénario du Grexit (...) (Le Figaro, 7/07/15, p. 3)

(17) Stopper le compte à rebours du Grexit, oui. (Le Figaro, 8/07/15, p. 2)

Rejoignant le potentiel/hypothétique, le sème du danger arrive en apport axiologique clairement négatif. Ainsi, le Grexit est catégorisé comme menace ou comme risque :

\footnotetext{
${ }^{5}$ Il s'agit de la BCE.
} 
(18) Deux semaines de négociations cruciales sous la menace de Grexit. (Le Figaro, $6 / 07 / 15$, pp. 4 et 5$)$

(19) (...) le risque d'une sortie de la Grèce de la zone euro est « réel ». (Le Monde, $7 / 07 / 15$, p. 1)

Remarquons que les deux noms déverbaux (« menace » et « risque ») permettent de ne pas exprimer les arguments sémantiques. L'agent et la cible de la menace restent dans l'oubli ainsi que l'objet du risque. Nous y reviendrons infra, car le gommage des arguments sémantiques nous semble s'inscrire dans une stratégie discursive propre à la description du Grexit.

Le discours d'information évoque aussi les conséquences - ou « l'engrenage », comme en (21) - d'un Grexit, et ces passages contiennent d'ailleurs du vocabulaire économique à proprement parler, comme «monnaie parallèle » (21), ou « liquidités » (23) :

(20) Même si la Bundesbank s'inquiète, selon le quotidien Handelsblatt, des conséquences d'un «Grexit» sur son propre bilan, les Allemands sont d'autant moins prêts à faire des concessions qu'ils sont convaincus qu'une sortie de ce pays de la zone euro aurait assez peu d'impact sur l'Allemagne. (Le Monde, 7/07/15, p. 3)

(21) Des faillites bancaires en série pourraient alors amorcer l'engrenage du Grexit : une économie frappée d'embolie, la brutale désillusion des Grecs, la création d'une monnaie parallèle, la sortie de l'euro. (Le Figaro, 7/07/15, p. 3)

(22) Le «Grexit » (...) une nécessité économique (...) une nécessité politique (Le Monde, $7 / 07 / 15$, p. 3)

(23) Le « Grexit » maintient les liquidités de la Grèce (Le Monde, 7/07/15, p.7)

(24) Les banques grecques au bord du gouffre (titre, Le Monde, 7/07/15, p. 7)

(25) La sortie de la Grèce de l'union monétaire (...) comparée à un Lehmann Brothers européen (Le Monde, 7/07/15, p. 7)

Comme nous l'avons remarqué supra, le Grexit ne renvoie pas directement à des événements réels (définis par Moirand, 2007 ou Veniard, 2013) comme bien d'autres dénominations ${ }^{6}$. Cet aspect particulier range le Grexit parmi les paroles construites en événement, un peu comme la tolérance zéro ou le Kärcher, parti du dire «nettoyer les cités au Kärcher » et ayant d'ailleurs donné lieu à des inventions lexicales du type Kärcher zéro ou l'immigration zéro (cf. à ce propos l'étude de Fred Hailon, 2014). De la sorte, le Grexit fonctionne souvent sur le mode de la modalisation autonymique ( $c f$. Authier-Revuz, 1992, p. 39) où le mot n'est pas un simple médiateur entre la réalité des signes et la réalité des choses, il s'impose comme objet du dire?

\footnotetext{
${ }^{6}$ Pour Tchernobyl c'est une catastrophe nucléaire connue, pour la guerre d'Afghanistan, un conflit militaire bien réel (cf. analyse de Veniard, 2009), ou pour le 11 septembre 2001, un attentat terroriste authentique.

${ }^{7}$ Il s'agit de modes complexes du dire qui cumulent deux opérations sémiotiques à la fois : dénotative, qui renvoie au monde, et métalinguistique, qui renvoie au signe.
} 
Les guillemets qui encadrent le vocable sont ainsi la trace d'une opération métalinguistique locale de " prise de distance » envers un mot et de «suspension de prise en charge » de ce mot par le locuteur (Authier, 1981, pp. 127-128). En voici des exemples :

(26) (...) la Grèce, privée de liquidités, devra, en urgence, pour éviter un effondrement total de son économie, en passer par une monnaie parallèle à l'euro, et ira tout droit au « Grexit ». (Le Monde, 8/07/15, p. 2)

(27) Ils ont oublié que Wolfgang Schäuble, le ministre allemand des Finances, appelait déjà à « Grexit ». (Libération, 6/07/15, p. 5)

(28) (...) éviter le « Grexit» (...) (Le Monde, 8/07/15, p. 1)

(29) Le compte à rebours avant le «Grexit », la sortie de la Grèce de la zone euro, a commencé. (Le Monde, 9/07/15, p. 1)

Le discours médiatique met en jeu ce côté métalinguistique du Grexit, exploitant une fonction magique du langage : le verbe y est omnipotent, avec son « abracadabra » ou toute autre formule (ici Grexit), l'énonciateur-magicien croit produire l'effet souhaité, et surtout, si on efface le mot, la réalité disparaitra quasi naturellement. De la sorte, au niveau des croyances discursives, la force surnaturelle de la nomination peut modifier le monde réel. Le mot «tabou» en apposition (30) qualifie le Grexit d'un interdit d'ordre culturel et/ou religieux qui pèse sur le langage (emploi des guillemets). La matérialité du signe s'impose également en (31), (32) et (33). Ces énoncés adoptent, pour ainsi dire, un point de vue du sorcier qui, par ses incantations, arrive à agir sur le réel :

(30) Tabou il y a encore un mois, le « Grexit », une sortie grecque de la zone euro (...) (Le Monde, 8/07/15, p. 5)

(31) (...) François Hollande refuse de prononcer l'expression « Grexit» mais le président de la République semble de plus en plus se préparer au pire des scénarios : la sortie programmée de la Grèce de la zone euro. (...) (Le Monde, 9/07/15, p. 3)

(32) Le mot « Grexit » n'est pas encore prononcé (Le Figaro, 7/07/15, p. 3)

(33) Le « Grexit » n'est pas une fatalité (Le Monde, 9/07/15, p. 15)

\section{ENJEUX DES REFORMULATIONS DU NDÉV «EXIT » / «SORTIE »}

Le nom d'événement (désormais $\mathrm{N}_{\text {dév }}$ ) « sortie » est au centre de la reformulation quasi littérale du Grexit (= 'la sortie de la Grèce de la zone euro', $c f$. infra). Trois aspects de cette reformulation retiendront notre attention dans cette section. Premièrement, il s'agit de l'expression de l'agent et des autres arguments sémantiques, en second lieu des essais de redéfinition du mot « sortie », et en troisième lieu de la sous-catégorisation abondante concernant le concept de sortie. La question de l'agentivité nous semble cruciale dans le discours à thématique socio-économique 
visant l'explication de différents dangers économiques. La redéfinition du nom noyau de l'expression examinée ainsi que sa sous-catégorisation diluent le sens «prédictible » du mot-valise examiné.

\subsection{OPACITÉ DE L’AGENTIVITÉ, GOMMAGE D’AUTRES ARGUMENTS}

Le mot-valise Grexit se prête avant tout à une explication analytique dévoilant simplement la combinatoire des éléments lexicaux qui le composent. Sur l'axe syntagmatique, cela donne, le plus souvent en apposition, la reformulation «une/la sortie grecque/de la Grèce de la zone euro », par exemple :

(34) Le compte à rebours avant le "Grexit », la sortie de la Grèce de la zone euro, a commencé. (Le Monde, 9/07/15, p. 1)

(35) Tabou il y a encore un mois, le «Grexit», une sortie grecque de la zone euro (...) (Le Monde, $8 / 07 / 15$, p. 5)

Le Grexit est donc reformulé par un syntagme nominal dont le noyau est un nom déverbal à statut transcatégoriel, qui présuppose l'existence d'un procès (produisant un « effet de préconstruit » selon Sériot (1986, p. 27) et qui présente des propriétés associées habituellement à des items prédicatifs qui peuvent exprimer des événements, réaliser des arguments (du point de vue syntaxique et sémantique) et marquer l'aspect (Knittel, 2015, pp. 10-14). Si la lecture de «sortie », exprimant un procès télique, ne peut être qu'événementielle, l'expression de l'agentivité, liée aux réalisations lexicales de l'argument « agent 》 ( "grecques » et « de la Grèce ») reste assez ambiguë. L'agentivité prototypique, considérée comme la cause ultime d'un événement, s'attribue à un être animé et particulièrement à un humain. L'agent se voit alors attribuer formellement une intention, un contrôle et une télicité pour les différentes actions qu'il exerce sur le monde extérieur (Bossong, 1998, p. 198). Le complément «de la Grèce» dans le syntagme «la sortie de la Grèce de la zone euro » (équivalant à « La Grèce sort/sortira de la zone euro ») exprime l'agent sur le mode métonymique : l'agent effectif supposé est remplacé par sa localisation spatiale. Ceci dit, nous partageons l'avis de Michelle Lecolle (2002, p. 44) que, dans la presse d'information, la métonymie entraîne toujours un certain flou référentiel: la référence paraît indéterminée et peu précise, le procès semble être contrôlé plutôt par des causes non-humaines. Il en va de même en (35) où le degré de contrôle du procès exprimé est minime car l'un des arguments est articulé par l'adjectif relationnel (grecque $=$ de la Grèce). Si l'on distingue entre l'agentivité et la causalité, le discours sur le Grexit, au niveau des opérations cognitives et énonciatives, navigue vers une agentivité non prototypique correspondant à la description instrumentale des événements ( $c f$. Charaudeau, 1997, p. 409 ; Veecock, 2012, pp. 117-150). La sortie de la zone euro semble donc ne pas être contrôlée par les êtres humains 
mais par des instruments ou par des causes non-humaines. Dans d'autres contextes encore, la Grèce évolue de cette agentivité affaiblie («la sortie de la Grèce ») à la passivité totale : «sortir la Grèce de l'euro » (36), « mettre dehors » (37), « chasser » (38), « expulser» (41):

(36) L'Europe prête à sortir la Grèce de l'euro (Le Figaro, 7/07/15, p. 1 [titre])

(37) « mettre la Grèce dehors » (Libération, 7/07/15, pp. 2 et 3)

(38) Car si la Grèce finit par être chassée de la zone euro (...) (Le Figaro, 6/07/15, p. 7)

(39) (...) le sommet européen de mardi soir pourrait acter la sortie d'Athènes de la monnaie unique. (Le Figaro, $7 / 07 / 15$, p. 1 [lead])

(40) (...) provoquer de facto un « Grexit » (...) (Le Monde, 8/07/15, p. 3)

(41) (...) un Grexit, lui, correspondrait à l'expulsion d'un pays au bord du défaut d'une union monétaire qui continuerait d'exister. (Le Monde, 8/07/15, p. 5)

(42) «Je suis contre le "Grexit", je veux l'éviter. Personne ne peut vouloir expulser les Grecs ». (Le Monde, $8 / 07 / 15$, p. 4)

Dans les cas « passifs » ci-dessus, l'argument « la Grèce » dans les reformulations du Grexit exprime l'objet du procès sans que son agent soit exprimé. Remarquons en outre que l'emploi des infinitifs n'y est pas anodin, car il gomme l'agentivité, comme le fait également l'emploi du passif sans complément d'agent en (38).

\subsection{AVATARS DISCURSIFS DU SENS LEXICAL DU N $\mathrm{DÉV}_{\text {« }}$ SORTIE »}

La part du variable dans ce discours socio-économique est importante : nombre de reformulations in praesentia modifient le sens lexical sur lequel s'appuie la dénomination. En voici des exemples :

(43) Le Grexit - sortie, congé ou quarantaine - (...) (Le Figaro, 7/07/15, p. 2)

(44) (...) « mettre la Grèce en congé de l'euro » (...) (Le Monde, 8/07/15, p. 4)

En (43), par l'ajout de noms dont l'un est juxtaposé et l'autre coordonné au lexème «sortie», ce dernier étant dépourvu du complément facultatif indiquant l'agent de l'action nominalisée, le Grexit s'explique par deux substantifs événementiels : " congé », lexème contenant toujours l'idée d'autorisation, et " quarantaine », qui n'est rien d'autre qu'un isolement imposé ( $c f$. Larousse en ligne). Ces reformulations apportent des modifications au profil lexical de Grexit en y introduisant, entre autres, les sèmes de [+ 'contrôle'] (un "congé » étant toujours autorisé) et de [+ 'injonction'] (la « quarantaine » étant une «mesure policière ») et affectent ainsi une opération énonciative très importante concernant l'attribution de l'agentivité. Le rôle de la Grèce dans l'actance du procès est finalement réduit à zéro, alors que l'agent présupposé effectif s'oublie et reste dans l'implicite du discours, car la réalisation des arguments pour des items prédicatifs comme « congé » ou « quarantaine » n'est que facultative. 
«La sortie » se reformule ensuite par « le départ» (47) et, sur le mode antinomique, par «une impasse» (45), et encore, sur le mode métaphorique, par «le divorce » (50) et «la séparation » (46), ouvrant ainsi de nouvelles classes référentielles :

(45) La sortie de l'euro de la Grèce est une impasse juridique (Le Monde, 9/07/15, p. 15 [titre])

\subsection{PROLIFÉRATION CATÉGORISATIONNELLE}

Si l'agentivité s'oublie ou reste dans l'implicite, le $\mathrm{N}_{\text {dév }}$ sortie reformulant exit est sous-catégorisé à plusieurs reprises et ouvre de nouvelles classes référentielles axiologiquement polarisées : il y a donc une «sortie désordonnée » $(48,49)$, reformulée aussi par «le chaos» (ce qui présuppose l'existence d'une sortie « ordonnée »), ensuite une « sortie temporaire », livrée avec une explication entre parenthèses ("pour réformer le pays »), qui préétablit «une sortie définitive ». Suit une/un "séparation/divorce/départ (à l') amiable », exprimant une voie de conciliation qui présuppose la voie contentieuse :

(46) (...) la séparation amiable, une sortie de la monnaie européenne, sortie « temporaire » (pour réformer le pays) (Libération, 7/07/15, pp. 2 et 3)

(47) Deuxième hypothèse : le départ à l'amiable, le «Grexit » (...) (Le Monde, 7/07/15, p. 3 [intertitre])

(48) Troisième hypothèse : la sortie désordonnée, le «Grexident » (Le Monde, 7/07/15, p. 3 [intertitre])

(49) (...) la sortie désordonnée (...) (Libération, $7 / 07 / 15$, pp. 2 et 3 )

(50) Trois scénarios possibles (...) reprise de négociations, divorce à l'amiable (le « Grexit ») ou sortie désordonnée de l'euro (le « Grexident », une sortie de la Grèce de la zone euro par accident (...) (Le Monde, 7/07/15, p. 3)

(51) (...) l'idée d'une sortie « ordonnée » de la Grèce de la zone euro (...) (Le Monde, $8 / 07 / 15$, p. 4)

(52) Le Grexit, résultat affiché ou simplement plausible (...) (Le Figaro, 7/07/15, p. 2)

(53) Le "Grexident», la faillite de la Grèce (reconnaissances de dettes peu à peu acceptées comme monnaie parallèle) (...) l'économie du pays (...) plongerait dans l'abîme. (Le Monde, 7/07/15, p. 3)

(54) (...) «Grexident », ce mot désignant une sortie de la Grèce de l'euro par accident (...) (Le Monde, $9 / 07 / 15$, p. 15)

(55) En attendant, le scénario du « Grexident », une sortie de la Grèce de la zone euro par accident (...) (Le Monde, 7/07/15, p. 3)

On voit que, du coup, le Grexit est catégorisé comme une sortie ordonnée, négociée par une voie de conciliation et opposée à un Grexident, défini dès lors comme « sortie de la Grèce par accident» et reformulé à son tour par « la faillite » (53). 


\section{CONCLUSION}

La part de l'implicite et du variable dans ce discours indécis construisant le sens social du Grexit est importante: de nombreuses nominalisations, les infinitifs, le passif sans complément d'agent gomment l'agent et/ou la cible de la prédication. La Grèce elle-même évolue de l'agentivité («la sortie de la Grèce») à la passivité ( «sortir la Grèce de l'euro »). Les causes non-humaines prévalent sur le monde économique, les décisions se prennent d'elles-mêmes. Le discours flotte dans le vague de différents univers lexicaux. Les reformulations du Grexit, variées et parfois contradictoires, contribuent à diluer son sens. Nous pensons que le discours d'information, même s'il relève de la thématique économique dont les concepts sont pointus, ne diffuse pas les dires experts dans le but d'expliquer quoi que ce soit, mais construit une sorte de récit médiatique approximatif qui socialise la conscience du risque économique. Évoquons à ce propos les calques formels du Grexit diffusés par les médias depuis un certain temps : le Brexit (littéralement = la sortie de la Grande Bretagne de la zone euro) et le Polexit (littéralement = la sortie de la Pologne de la zone euro). Or, leurs emplois ne correspondent pas du tout au sens du vocable de départ, issu du discours économique, puisque ni la Grande Bretagne, ni la Pologne n'ont jamais fait partie de la zone euro. On voit que le sens compositionnel de la formule, « prédictible » à partir des sens des éléments composant le mot-valise, n'est pas forcément référentiel en discours, et n'est pas forcément non plus obligatoire dans la formation des vocables Brexit et Polexit. Le Grexit semble condenser une problématique qui touche moins à la connaissance encyclopédique des individus et plus à une configuration d'énoncés en discours. On voit que le langage lui-même a des ressources interprétatives. Le Grexit est un véritable événement discursif, le discours le rend intelligible dans une opération d'interprétation et permet de le comprendre. Autement dit, le Grexit est mis en récit, il est une construction médiatique qu'il ne faut pas confondre avec la construction de la réalité. 


\section{BIBLIOGRAPHIE}

Authier, J. (1981). Paroles tenues à distance. In B. Conein, J. J. Courtine, F. Gadet, \& M. Pêcheux (éds), Matérialités Discursives (pp. 27-142). Lille : PUL.

Authier-Revuz, J. (1992). Repères dans le champ du discours rapporté. L'information grammaticale, 55 (pp. 38-42). Peeters Publishers. Retrieved from https://doi.org/10.3406/igram.1992.3186

Bossong, G. (1998). Le marquage différentiel de l'objet dans les langues d'Europe. In J. Feuillet (éd.), Actance et valence dans les langues de l'Europe (pp. 193-258). Berlin : Mouton de Gruyter. Retrieved from https://doi.org/10.1515/9783110804485.193; https://doi.org/10.1515/9783110804485.259

Buiter, W. H., Rahbari, E. (2012). Global Economics View. Rising Risks of Greek Euro Area Exit. Retrieved from http://willembuiter.com/grexit.pdf

Calabrese-Steimberg, L. (2010). Le réemploi de dénominations d'évènements dans la construction d'évènements prototypiques. Communications $d u I V^{e}$ Ci-dit. Retrieved from http://revel.unice.fr/ symposia/cidit/index.html?id $=398$

Dendale, P. (2001). Les problèmes linguistiques du conditionnel français. In P. Dendale et L.Tasmowski (éds), Le conditionnel en français (Recherches linguistiques, 25, pp. 7-18). Paris : Klincksieck.

Détrie, C., Siblot, P., Verine, B. (éds) (2001). Termes et concepts pour l'analyse du discours. Une approche praxématique. Paris : Champion.

Hailon, F. (2014). Contribution à une sémantique médiatique numérique (nouvelles pratiques médiatiques numériques et savoirs de sens commun). Studii de Lingvistică, 4, pp. 93-109.

Kleiber, G. (1997). Sens, référence et existence : que faire de l'extra-linguistique ? Langages, 127 (Langue, praxis et production de sens), pp. 9-37. DOI : 10.3406/lgge.1997.2123

Knittel, M. L. (2015). La nominalisation : un état des lieux. Le Français Moderne, 1, pp. 3-17.

Krieg-Planque, A. (2003). "Purification ethnique». Une formule et son histoire. Paris : CNRS. Retrieved from https://doi.org/10.4000/books.editionscnrs.5392

Krieg-Planque, A. (2006). «Formules » et « lieux discursifs ». Propositions pour l'analyse du discours politique. Semen, 21, pp. 19-47.

Krieg-Planque, A. (2009). La notion de "formule » en analyse du discours. Cadre théorique et méthodologique. Besançon : Presses universitaires de Franche-Comté.

Krieg-Planque, A. (2010). La formule « développement durable». Un opérateur de neutralisation de la conflictualité. Langage et société, 134, pp. 5-29. Retrieved from https://doi.org/10.3917/ls. 134.0005

Lecolle, M. (2002). Personnifications et métonymies dans la presse écrite : comment les différencier? Semen, 15. Retrieved from http://semen.revues.org/2396

Moirand, S. (2007). Le discours de la presse quotidienne. Observer, analyser, comprendre. Paris : Presses Universitaires de France.

Mortureux, M.-F. (1993). Paradigmes désignationnels. Semen, 8. Retrieved from http://semen.revues. org $/ 4132$

Paveau, M.-A. (2008). Le toponyme, désignateur souple et organisateur mémoriel. L'exemple du nom de bataille. Mots. Les langages du politique, 86. DOI : 10.4000/mots. 13102

Searle, J. R. (1972). Les actes de langage. Paris : Hermann.

Seriot P. (1986). Langue russe et discours politique soviétique : analyse des nominalisations. Langages, 81 (Analyse de discours, nouveaux parcours [Hommage à Michel Pêcheux]), pp. 11-41. Retrieved from https://doi.org/10.3406/lgge.1986.2476

Siblot, P. (1997). Nomination et production de sens : le praxème. Langages, 127, pp. 38-55. Retrieved from https://doi.org/10.3406/lgge.1997.2124

Siblot, P. (2001). De la dénomination à la nomination. Cahiers de praxématique, 36, pp. 189-214. 
Veecock, C. (2012). Agentivité, modalitées de contrôle et subjectivité. Linguistique. Université Michel de Montaigne - Bordeaux III, Français. Retrieved from https//www. archives-ouvertes.fr.

Veniard, M. (2009). La dénomination propre « la guerre d'Afghanistan » en discours : une interaction entre sens et référence. Les Carnets du Cediscor, 11. Retrieved from http://cediscor.revues.org/753

Veniard, M. (2013). La nomination des événements dans la presse. Essai de sémantique discursive. Besançon : Presses Universitaires de Franche-Comté. 